\title{
English Translation and Validation of the Zurich Chronic Middle Ear Inventory (ZCMEI-21-E) Assessing Quality of Life in Chronic Otitis \\ Media: A Prospective International Multicenter Study
}

Running title: ZCMEI-21 English translation and validation

\section{Authors:}

Michail Chatzimichalis (1), Lorenz Epprecht (2, 3), Stefan Weder (4, 5), Chanan Shaul (4), Kristi J. Engle Folchert (6), Maria C. Machala (6), Madeline M. Goosmann (7, 8), Marc Naville (3), Angela Zhu (3), Christof Röösli (7, 8), Daniel J. Lee (2, 3), Stephen P. Cass (6), Robert Briggs (4), Alexander M. Huber $(7,8)$, David Bächinger $\left(7,8,{ }^{*}\right)$

\section{Affiliations:}

(1) Dorset County Hospital, Dorchester, UK

(2) Department of Otolaryngology - Head and Neck Surgery, Massachusetts Eye and Ear Infirmary, Boston, MA, USA

(3) Eaton-Peabody Laboratories, Massachusetts Eye and Ear Infirmary, Boston, MA, USA

(4) Department of Surgery, Otolaryngology, University of Melbourne, East Melbourne, Victoria, Australia

(5) Department of Otolaryngology, Head and Neck Surgery, University Hospital Bern, Bern, Switzerland

(6) Department of Otolaryngology, University of Colorado Anschutz Medical Campus, Aurora, Colorado, USA

(7) Department of Otorhinolaryngology, Head and Neck Surgery, University Hospital Zurich, Zurich, Switzerland

(8) University of Zurich, Zurich, Switzerland

\section{* corresponding author:}

David Bächinger, david.baechinger@usz.ch, telephone +4144 2555850

\section{Conflict of interest}

All authors declare that they have no conflict of interest.

This is the author manuscript accepted for publication and has undergone full peer review but has not been through the copyediting, typesetting, pagination and proofreading process, which may lead to differences between this version and the Version of Record. Please cite this article as doi: $\underline{10.1111 / \text { coa. } 13275}$ 


\section{Acknowledgements}

None.

\section{ABSTRACT}

Objectives: To translate and validate the Zurich Chronic Middle Ear Inventory (ZCMEI21) for the English language in order to provide an English instrument to assess healthrelated quality of life in chronic otitis media (COM).

Design: Pilot translation study including cognitive debriefings, prospective multicenter cross-sectional psychometric validation study.

Setting: Four tertiary referral centers in three different English-speaking countries (United Kingdom, United States of America and Australia).

Participants: Adult patients suffering from COM.

Main outcome measures: The English translation of the ZCMEI-21 (ZCMEI-21-E) and the five-level version of the EQ-5D questionnaire. The EQ-5D, which constitutes a generic measure of health-related quality of life, consists of a descriptive system score and a visual analogue scale. Statistical outcomes included single item descriptive statistics, internal consistency (Cronbach's $\alpha$ ) as an indicator of reliability, as well as construct validity.

Results: A total of 124 patients suffering from COM were included. The mean age was 50.1 years (SD 16.9 years) and 72 (58.1\%) were males. The Cronbach's $\alpha$ of the ZCMEI21-E was 0.91, suggesting an excellent internal consistency. The Spearman's correlation coefficient of the ZCMEI-21-E total score was $0.55(p<0.0001)$ for convergent construct validity with EQ-5D descriptive system score and $0.57(p<0.0001)$ with the EQ-5D visual analogue scale.

Conclusions: The ZCMEI-21-E is a new validated questionnaire that provides clinicians with a short, comprehensive and reliable instrument to quantify health-related quality of life in patients suffering from COM. The ZCMEI-21-E may be of use in clinical routine as well as in outcome research and monitoring.

\section{KEYWORDS}

Otology, chronic otitis media, cholesteatoma, electronic patient-reported outcome measure, questionnaire, HRQoL, ZCMEI-21, English, translation 


\section{KEY POINTS}

- The ZCMEI-21-E is a new validated English instrument to quantify health-related quality of life in patients suffering from chronic otitis media.

- The ZCMEI-21-E is a short, comprehensive and reliable instrument that may be of use in clinical routine as well as in outcome research and monitoring.

- As the ZCMEI-21-E will become available in multiple languages, it will be possible to compare study results conducted in different countries, which is a step forward towards an international standardization of reporting HRQoL in COM.

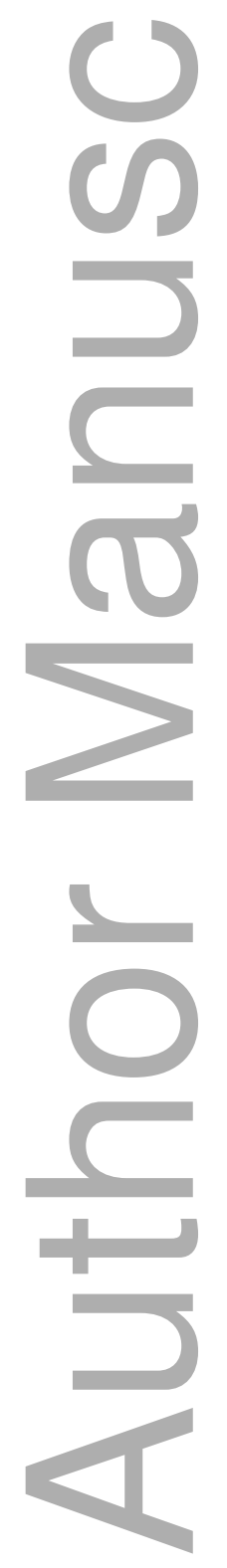


DR DAVID BÄCHINGER (Orcid ID : 0000-0002-6336-494X)

Article type _ : Original Manuscript

\section{ABSTRACT}

Objectives: To translate and validate the Zurich Chronic Middle Ear Inventory (ZCMEI21) for the English language in order to provide an English instrument to assess healthrelated quality of life in chronic otitis media (COM).

Design: Pilot translation study including cognitive debriefings, prospective multicenter cross-sectional psychometric validation study.

Setting: Four tertiary referral centers in three different English-speaking countries (United Kingdom, United States of America and Australia).

Participants: Adult patients suffering from COM.

Main outcome measures: The English translation of the ZCMEI-21 (ZCMEI-21-E) and the five-level version of the EQ-5D questionnaire. The EQ-5D, which constitutes a generic measure of health-related quality of life, consists of a descriptive system score and a visual analogue scale. Statistical outcomes included single item descriptive statistics, internal consistency (Cronbach's $\alpha$ ) as an indicator of reliability, as well as construct validity.

Results: A total of 124 patients suffering from COM were included. The mean age was 50.1 years (SD 16.9 years) and 72 (58.1\%) were males. The Cronbach's $\alpha$ of the ZCMEI21-E was 0.91, suggesting an excellent internal consistency. The Spearman's correlation coefficient of the ZCMEI-21-E total score was 0.55 ( $p<0.0001)$ for convergent construct validity with $E Q-5 D$ descriptive system score and $0.57(p<0.0001)$ with the $E Q-5 D$ visual analogue scale.

Conclusions: The ZCMEI-21-E is a new validated questionnaire that provides clinicians with a short, comprehensive and reliable instrument to quantify health-related quality of life in patients suffering from COM. The ZCMEI-21-E may be of use in clinical routine as well as in outcome research and monitoring.

\section{KEY POINTS}


- The ZCMEI-21-E is a new validated English instrument to quantify health-related quality of life in patients suffering from chronic otitis media.

- The ZCMEI-21-E is a short, comprehensive and reliable instrument that may be of use in clinical routine as well as in outcome research and monitoring.

- As the ZCMEI-21-E will become available in multiple languages, it will be possible to compare study results conducted in different countries, which is a step forward towards an international standardization of reporting $\mathrm{HRQoL}$ in COM.

\section{INTRODUCTION}

In clinical outcome research, patient-reported (subjective) outcomes have become important endpoints in addition to the traditional, objective endpoints. ${ }^{1}$ One crucial patientreported outcome is health-related quality of life (HRQoL), which reflects the subjective disease burden. HRQoL is a complex construct that is usually measured by patientreported outcome measures (PROMs), e.g. questionnaires. These questionnaires may be either generic or disease-specific, but since HRQoL is often influenced by specific symptoms, generic questionnaires are usually not adequate to reliably detect all facets of HRQoL. $^{2}$

During the last two decades, assessment of HRQoL in chronic otitis media (COM) has gained increasing attention. ${ }^{3}$ In COM, HRQoL is affected by many factors, including hearing loss as well as other ear symptoms including discharge, tinnitus, vertigo, and balance problems. ${ }^{4}$ Furthermore, patients suffering from COM may experience anxiety, depression, or social isolation due to their ear disease. ${ }^{2,4,5}$ As generic questionnaires have been deemed unsuitable for reliable detection of various aspects of HRQoL in $\mathrm{COM}^{2,6}$, disease-specific questionnaires have been developed for several languages (reviewed in ${ }^{3}$ and ${ }^{7}$ ).

For the English language, the first COM-specific instrument developed was the Chronic Ear Survey $\left(\mathrm{CES}^{8}\right)$ followed by the Chronic Otitis Media Questionnaire (COMQ-12 ${ }^{9}$ ). The COMQ-12 was then further developed into the Chronic Otitis Media Benefit Inventory $\left(\mathrm{COMBI}^{10}\right)$, which dynamically assesses of HRQoL in COM. However, these questionnaires have some shortcomings, which are a primary focus on disease specific health and a strong focus on the physical dimension, as previously discussed. ${ }^{11}$ To further improve the assessment of HRQoL in COM, we recently developed a new questionnaire, the Zurich Chronic Otitis Media Outcome Inventory (ZCMEI-21). ${ }^{11}$ The ZCMEI-21 aims to cover the physical dimension, but also to specifically assess essential components of HRQoL, e.g. anxiety or social isolation., ${ }^{2,511}$ The ZCMEI-21 comprises 21 questions 
("items"), which are grouped in four subscales: I. ear signs and symptoms, II. hearing, III. psychosocial impact, and IV. medical resources. The questions are answered by use of a 5-point Likert scale ranging from no emotional/physical impact (coded as 0 ) to severe emotional/physical impact (coded as 4). The ZCMEI-21 has been originally developed and validated for the German language. In an effort to make the ZCMEI-21 available in other languages to provide a comparable and consistent assessment and reporting of HRQoL in COM, several translation and validation studies have been initiated. ${ }^{12,13}$ In the present study, we aimed to translate the ZCMEI-21 into English and validate the translation for measuring $\mathrm{HRQ}$ oL with COM.

\section{PATIENTS AND METHODS}

\section{Ethical considerations}

The study protocol was approved by each local Ethics Committee in accordance with the Helsinki declaration. Informed consent was obtained from all the participants.

\section{Patients and study centers}

We recruited patients with either form of COM, i.e. with cholesteatoma (otitis media chronica cholesteatomatosa [OMCC]) or without cholesteatoma and a chronic tympanic membrane perforation (otitis media chronica simplex [OMCS]) from four study centers. The study centers consisted of (i) the Dorset County Hospital (Dorchester, UK), (ii) the Department of Otolaryngology - Head and Neck Surgery at the Massachusetts Eye and Ear Infirmary (Boston, MA, USA), (iii) the Department of Otolaryngology, University of Melbourne (East Melbourne, Victoria, Australia), and (iv) the Department of Otolaryngology of the University of Colorado Hospital (Aurora, CO, USA). The latter institution used Research Electronic Data Capture (REDCap) to collect and manage study data ${ }^{14}$, the other institutions used Microsoft Excel (Microsoft Corp., Redmond, WA, USA). The detailed study design is described in Figure 1.

\section{Translation process}

We followed a standardized approach for the translation and cultural adaption of questionnaires according to the "Principles of Good Practice for the Translation and Cultural Adaptation Process for Patient-Reported Outcomes Measures" according to the International Society for Pharmacoeconomics and Outcomes Research Task Force 
(Figure 1). ${ }^{15}$ In brief, two independent professional translation agencies each developed a forward translation. Then, native English-speaking physicians merged these two translations into a single translation according to their preference concerning the appropriate wording of each question (ZCMEI-21-E, v1). The first version was tested in five patients to highlight difficulties in comprehension of the questions and to identify any items that may be conceptually inappropriate (first cognitive debriefing). The feedback of the patients and the involved professionals was the basis for modifications, resulting in the second version (ZCMEI-21-E, v2). Subsequently, this version was back translated from a third professional translation agency, reviewed against the original German version and modified ensuring conceptual equivalence of the translation (ZCMEI-21-E, v3). The second cognitive debriefing that followed led to no further modifications. The final version of the questionnaire (ZCMEI-21-E) was used for the validation process. The ZCMEI-21-E is available as supplementary electronic file (ZCMEI-21-E.pdf).

\section{Validation process}

\section{Assessment instruments}

We administered the ZCMEI-21-E developed as described above. We added a question addressing HRQoL directly in order to assess construct validity, similar to the study validating the original version of the ZCMEI. ${ }^{11}$ ZCMEI-21-E total scores were calculated by summing up the single item scores (range $0-4$ ) resulting in a maximum total score of 84. A higher ZCMEI-21 total score corresponds to a worse HRQoL. In addition to the ZCMEI-21-E, the five-level version of the EQ-5D questionnaire (EQ-5D-5L, hereinafter referred to as EQ-5D) was administered..$^{16}$ The EQ-5D was used as a generic measure of health outcome since this questionnaire is well-established for this use. The EQ-5D questionnaire consists of five questions and a visual analogue scale (VAS). The five questions cover mobility, self-care, usual activities, pain/discomfort, and anxiety/depression. The answers to these questions yield an index value (descriptive system score), which ranges from 0 to 1 ( 1 corresponding to a perfect HRQoL). The VAS ranges from 0 to 100 (100 corresponding to the best health state).

\section{Statistical analysis}

All statistical tests were selected before data collection. Statistical analyses were performed using IBM SPSS Statistics for Windows (version 25, IBM Corp., Armonk, NY, USA) and Prism (version 7, GraphPad Software, La Jolla, CA, USA). The significance 
level was set to $p<0.05$. Values are reported as mean $\pm S D$ or as absolute number and percentage. Item total-correlation was calculated to assess if an item correlates with the total score. An Item total-correlation $\geq 0.3$ indicates that a question correlates well with the total score and represents one criterion for a strong item. For assessing reliability of the ZCMEI-21-E, internal consistency as an indicator of reliability was determined by the use of Cronbach's $\alpha$. Cronbach's $\alpha \geq 0.7$ was considered as acceptable. ${ }^{17}$ Frequency distribution was analyzed by (i) inspection of the histogram and (ii) statistical normality tests (D'Agostino-Pearson normality test, $p>0.05$ indicates normal distribution). Construct validity was determined using a question (question 22, added to the ZCMEI21-E) that directly assessed HRQoL. Furthermore, total and sub-scores of the ZCMEI-21$E$ and EQ-5D-5L descriptive system and VAS scores were correlated using Spearman's rank correlation and linear regression analysis including mean prediction intervals. For the validation study, a minimum sample size of 110 was calculated. This was based on a subject to item ratio of $5: 1$ and a total of 22 items, including 21 items of the questionnaire and one added item for validation purposes. ${ }^{18}$

\section{RESULTS}

A total of 124 patients were included in the present study. (Dorset County Hospital, Dorchester, UK: $n=26$; Department of Otolaryngology - Head and Neck Surgery, Massachusetts Eye and Ear Infirmary, Boston, USA: $\mathrm{n}=29$; Department of Otolaryngology, University of Melbourne, Australia: $n=39$; Department of Otolaryngology, University of Colorado Hospital, USA: $n=30$ ). The mean age was $59.1 \pm 16.9$ years. OMCC was present in 53 (42.8\%) patients and OMCS was present in 66 (53.2\%) patients. Both forms in separate ears were present in $5(4.0 \%)$ patients. At time of completion of the ZCMEI-21-E, $82(66.1 \%)$ patients had already undergone surgery for COM. Complete demographics and patient characteristics are provided in Table 1.

Concerning single item statistics, we found well distributed answers and the full range of answers used in every question (Table 2). Item-total-correlations of all items were well above 0.3 , with the exception of the last question that assessed the use of ear drops (Table 2).

For assessing the questionnaire's reliability, Cronbach's a as a measure of internal consistency was determined. We found a Cronbach's a of 0.91 for the entire questionnaire, which indicates an excellent internal consistency. Moreover, sufficient to 
excellent internal consistency was also found for the individual subscales (Table 2). Further, distribution of ZCMEI-21-E total scores was investigated. Both the inspection of the histogram (Figure 2A) and the D'Agostino-Pearson normality test $(p=0.10)$ suggested a Gaussian distribution. No significant differences in ZCMEI-21-E total scores was observed among the three different countries, i.e. the US, UK and Australia $(p=0.67, F$ $(2,605)=0.407$; two-way ANOVA; Figure S1A).

Comparing total scores among subgroups of the study population, we observed no significant differences among the mean total scores neither when patients were grouped according to the COM subtype nor according to having had surgery before (Figure 2B-C). Yet, patients with bilateral COM had a significantly higher mean ZCMEI-21-E total score than patients with unilateral $\operatorname{COM}(p=0.01$; Figure $2 D)$. A post hoc analysis using a conservative statistical approach with multiple t-tests and Bonferroni-correction revealed that patients suffering from bilateral disease had a significantly higher mean score in subscale III (psychosocial impact; $p=0.003$; Figure S1B). Further, statistically significantly higher single item scores of patients suffering from bilateral disease were found in questions 17 (adjusted $p=0.003$ ), 18 (adjusted $p=0.03$ ) and 19 (adjusted $p=$ 0.03; Figure S1C).

Next, the absolute ZCMEI-21 and EQ-5D scores and their correlations were compared between the original validation study ${ }^{11}$ and the ZCMEI-21-E (Table 3). Highly similar mean values and standard deviations for total scores, subscale scores and correlation coefficients were observed. A strong correlation between the ZCMEI-21-E total score and the question directly assessing HRQoL (added $22^{\text {nd }}$ question) was found $(r=0.67, p<$ 0.0001; Figure $3 \mathrm{~A}$ ). A highly significant albeit weaker correlation was observed between the ZCMEI-21-E total score and the EQ-5D descriptive system score $(r=0.55, p<$ 0.0001; Figure 3B) and the EQ-5D VAS $(r=0.57, p<0.0001$; Figure $3 C)$.

Lastly, the ZCMEI-21-E subscale scores were correlated to the EQ-5D descriptive system and VAS scores. Subscale I (ear signs and symptoms), subscale II (hearing) and subscale III (psychosocial impact) were moderately correlated to the EQ-5D descriptive system scores (Figure 4A-C) as well as to the EQ-5D VAS scores (Figure 4D-E), while all correlation coefficients were statistically significant $(p<0.0001$ for all correlation coefficients).

\section{DISCUSSION}




\section{Main findings}

We translated the ZCMEI-21 into English according to international guidelines. Next, we validated the ZCMEI-21-E by assessing measures for its reliability and validity. Cronbach's $\alpha$ of the total and subscale scores indicated excellent internal consistency, thus providing evidence for the ZCMEI-21-E's reliability. Assessing concurrent validity, we found that ZCMEI-21-E total scores correlated strongly with the answer to a question directly assessing HRQoL in COM. Furthermore, the ZCMEI-21-E total and subscale score significantly correlated with the EQ-5D descriptive system score and VAS. Yet, the latter correlations were weaker as compared to correlation to the question directly assessing HRQOL in COM. This indicates that, on the one hand, the ZCMEI-21-E measures the construct of $\mathrm{HRQoL}$, but, on the other hand, further corroborates the finding that measuring $\mathrm{HRQoL}$ in specific conditions, such as COM, should not be performed by use of generic questionnaires. ${ }^{2,6}$

\section{Results in context of previous research}

Besides the ZCMEI-21-E, other English instruments have been developed and validated for the use of measuring HRQoL in COM. ${ }^{8,19}$ However, the ZCMEI-21-E differs in several aspects from the preexisting English questionnaires. The CES developed in 2000 by Nadol et al. primarily assesses ear- and disease-specific health. ${ }^{8}$ Several questions of the CES have been adapted for the ZCMEI-21. The CES does not contain any questions covering vertigo/balance problems and tinnitus, both of which are considered as relevant symptoms of COM that possibly hamper HRQoL. ${ }^{11,20}$ Also, the CES does not provide a comprehensive assessment of psychosocial problems. In 2014, the COMQ-12 was developed by Philipps et al. aiming to measure HRQoL in COM. Although this questionnaire provides a more complete assessment of HRQoL than the CES, several shortcomings have been discussed previously. ${ }^{11}$ First, the COMQ-12 does not include questions regarding activity restriction due to COM, which is a focus of the CES and which is also included in the ZCMEI-21-E. Secondly, the COMQ-12 contains two questions regarding "lifestyle and work impact", but does not provide a broader assessment of psychosocial problems associated with COM. Lastly, both the CES and the COMQ-12 have a recall period of 6 months, which is markedly longer than the 2-week recall period of the ZCMEI-21-E. Although 2 weeks may seem a rather short recall period for a chronic condition, there are several advantages with recall periods covering weeks rather than months. These shorter recall substantially increase the accuracy of the 
recalled information provided as answers to the questionnaire ${ }^{21}$. Furthermore, a shorter recall period may render the ZCMEI-21-E more sensitive to changes, especially during the perioperative period. ${ }^{11}$ Taken together, the ZCMEI-21-E may offer several advantages over the existing English instruments assessing HRQoL in COM.

An interesting although fairly evident finding in our study was the significantly higher mean ZCMEI-21-E total score in patients with bilateral COM compared to patients with unilateral COM. This may indicate that patients suffering from bilateral COM have a worse HRQoL compared to patients who are unilaterally affected. Similar results have been previously obtained in a Turkish cohort. ${ }^{22}$ Yet, the latter results should be interpreted with care as the questionnaire used in this study was a translated version of the German Chronic Otitis Media Outcome Test (COMOT-15), which has never been validated for the Turkish language. ${ }^{7,20}$ The finding of worse HRQoL in bilateral COM may warrant further investigation beyond our current study.

\section{Limitations}

The present study used a highly heterogeneous COM cohort to validate the ZCMEI-21-E, which may impair the ability to make distinct statements regarding the specific application of the ZCMEI-21-E in COM subgroups, such as operated and non-operated patients or COM with and without cholesteatoma. However, we explicitly aimed to develop a questionnaire which can be applied to every patient suffering from COM regardless of status of surgery or type of COM. Thus, with the present data, the application of the ZCMEI-21-E is justified in all COM patients, but further studies may address differences in COM subgroups.

Furthermore, we used a paper-based version of the ZCMEI-21-E, while the original ZCMEI-21 has been developed as an electronic questionnaire, which can be delivered on a mobile phone or tablet computer. However, it has been demonstrated that results obtained by paper-based or electronic application of a questionnaire are highly similar [29]. Therefore, based on our present data, we propose that both the paper-based as well as electronic versions of the ZCMEI-21-E may be used in the future.

\section{Clinical implication and applicability for research and practice}

There is an increasing demand for physicians and scientists to collect patient-reported outcomes such as HRQoL on a routine basis in clinical routine as well as in outcome research and monitoring. ${ }^{23}$ The $\mathrm{ZCMEI}-21$ was developed for this purpose and is now 
available as a validated English version, the ZCMEI-21-E. The ZCMEI-21-E is a questionnaire, which is simple to apply and contains easily understandable questions while still providing a comprehensive assessment of HRQoL in COM. ${ }^{11}$ These data on HRQoL are a valuable completion of the traditional, objective COM outcomes, such as audiometry and recurrence rate. ${ }^{1}$ Moreover, the availability of this instrument in different languages may increase the standardization of reporting HRQoL in COM.

\section{Conclusion}

In this study, we translated the ZCMEI-21 into English according to international guidelines. Subsequently, we conducted a prospective multicenter study including 124 COM patients. We collected sufficient data on reliability and validity of the ZCMEI-21-E to justify the application of the ZCMEI-21-E to measure HRQoL in English-speaking patients with COM in clinical routine and research.

\section{SUPPLEMENTARY MATERIAL}

\section{ZCMEI-21-E.pdf}

\section{REFERENCES}

1 Black N. (2013) Patient reported outcome measures may transform healthcare. Br. Med. J. 346, 19-21.

2 Guyatt G.H., Bombardier C. \& Tugwell P.X. (1986) Measuring disease-specific quality of life in clinical trials. Can. Med. Assoc. J. 134, 889-895.

3 Phillips J.S. \& Yung M.W. (2016) A systematic review of patient-reported outcome measures for chronic suppurative otitis media. Laryngoscope 126, 1458-1463.

4 Bakir S., Kinis V., Bez Y., et al. (2012) Mental health and quality of life in patients with chronic otitis media. Eur Arch Otorhinolaryngol 270, 521-526.

5 Mulrow C.D., Aguilar C., Endicott J.E., et al. (1990) Quality-of-life changes and hearing impairment: A randomized trial. Ann. Intern. Med. 113, 188-194.

6 Baumann I., Gerendas B., Plinkert P.K., et al. (2011) General and disease-specific quality of life in patients with chronic suppurative otitis media--a prospective study. Health Qual. Life Outcomes.

7 Lailach S., Baumann I., Zahnert T., et al. (2018) Aktueller Stand der Lebensqualitätsmessung bei Patienten mit chronischer Otitis media und Schallleitungsschwerhörigkeit. HNO 66, 578-589.

8 Nadol Jr. J.B., Staecker H. \& Gliklich R.E. (2000) Outcomes assessment for chronic otitis 
media: the Chronic Ear Survey. Laryngoscope 110, 32-35.

$9 \quad$ Phillips J.S., Haggard M. \& Yung M. (2014) A New Health-Related Quality of Life Measure for Active Chronic Otitis Media (COMQ-12). Otol. Neurotol. 35, 454-458.

10 Phillips J.S., Haggard M., Spencer H., et al. (2017) The Chronic Otitis Media Benefit Inventory (COMBI). Otol. Neurotol. 89, 1.

11 Bächinger D., Röösli C., Ditzen B., et al. (2016) Development and validation of the Zurich chronic middle ear inventory (ZCMEI-21): an electronic questionnaire for assessing quality of life in patients with chronic otitis media. Eur. Arch. Oto-Rhino-Laryngology.

12 Bächinger D., Takagi D., Yamada H., et al. (2018) Japanese translation, cross-cultural adaption and multicentre validation of the Zurich chronic middle ear inventory (ZCMEI-21Jap). Auris Nasus Larynx.

13 Ralli M., Quaranta N., Canale A., et al. (2018) Cross-cultural Adaption and Validation of the Zurich Chronic Middle Ear Inventory Translated Into Italian (ZCMEI-21-It)—a Prospective Multicenter Study. Otol. Neurotol.

14 Harris P.A., Taylor R., Thielke R., et al. (2009) Research electronic data capture (REDCap)-A metadata-driven methodology and workflow process for providing translational research informatics support. J. Biomed. Inform.

15 Wild D., Grove A., Martin M., et al. (2005) Principles of good practice for the translation and cultural adaptation process for patient-reported outcomes (PRO) measures: Report of the ISPOR Task Force for Translation and Cultural Adaptation. Value Heal. 8, 94-104.

16 Rabin R. \& de Charro F. (2001) EQ-5D: a measure of health status from the EuroQol Group. Ann. Med. 33, 337-43.

17 Cronbach L.J. (1951) Coefficient alpha and the internal structure of tests. Psychometrika 16, 297-334.

18 Anthoine E., Moret L., Regnault A., et al. (2014) Sample size used to validate a scale: a review of publications on newly-developed patient reported outcomes measures. Health Qual. Life Outcomes 12, 176.

19 Phillips J.S. \& Yung M.W. (2014) COMQ-12 scores in adult patients without chronic middle ear disease. Clin. Otolaryngol. 39, 362-367.

20 Baumann I., Kurpiers B., Plinkert P.K., et al. (2009) Development and validation of the Chronic Otitis Media Outcome Test 15 (COMOT-15). Measurement of health-related quality of life in patients with chronic otitis media. HNO 57, 889-895.

21 Stull D.E., Leidy N.K., Parasuraman B., et al. (2009) Optimal recall periods for patientreported outcomes: challenges and potential solutions. Curr. Med. Res. Opin. 25, 929942.

22 Demir U.L., Akyildiz M.Y. \& Alpay M. (2012) The Factors which Affect Disease-Specific Quality of Life in Patients with Chronic Otitis Media. Int. Adv. Otol 90, 371-378. 
Alakärppä A. \& Alho O.-P. (2012) Patient-recorded outcomes and quality of life in evidence-based medicine databases on most common ear, throat and nose procedures: a systematic review. Clin. Otolaryngol. 37, 436-45.

\section{FIGURE LEGENDS}

Figure 1. Study design for the English translation of the ZCMEI-21 (ZCMEI-21-E) and validation of the ZCMEI-21-E.

Figure 2. A ZCMEI-21-E total score frequency distribution with best-fitting Gaussian curve (bin width on x-axis: 4). B Comparison of ZCMEI-21-E total scores among patients grouped according to type of COM. OMCC, otitis media chronica cholesteatomatosa; OMCS, otitis media chronica simplex. One-way ANOVA with Tukey's range test as posthoc multiple comparison procedure. C Comparison of ZCMEI-21-E total scores in operated and non-operated patients. Unpaired t-test. D Comparison of ZCMEI-21-E total scores in patients suffering from unilateral and bilateral COM. Unpaired t-test. Whiskers indicate standard deviation range, bold horizontal line represents mean. N. s., not significant.

Figure 3. A Correlation of ZCMEI-21-E total scores and the question directly assessing HRQoL (added $22^{\text {nd }}$ question). 0 on $x$-axis corresponds to no impact on HRQoL in question 22; 4 on the $x$-axis corresponds to large impact on HRQoL in question 22. Spearman's rank correlation. B Spearman's rank correlation between ZCMEI-21-E total score and EQ-5D descriptive systems score. C Spearman's rank correlation between ZCMEI-21-E total score and EQ-5D VAS. Solid line indicates linear regression line, dashed lines indicate 95\% prediction interval; r, Spearman's rank correlation coefficient.

Figure 4. Spearman's rank correlation between ZCMEI-21-E subscale scores and EQ5D-5L (descriptive system scores and VAS). Solid line represents linear regression line, dashed lines represent 95\% prediction interval; r, Spearman's rank correlation coefficient.

Figure S1. A ZCMEI-21-E subscale and total scores in patients recruited in different countries. B ZCMEI-21-E subscale scores for patients suffering from unilateral and bilateral disease. C ZCMEI-21-E single item scores for patients suffering from unilateral and bilateral disease. ${ }^{*} p<0.05,{ }^{* *} p<0.01$, mean and standard deviation. 
Table 1. Demographics and clinical characteristics of the patients of the cohort in which the ZCMEI-21-E was validated.

\begin{tabular}{ll}
\hline & Validation cohort $(\mathbf{n}=\mathbf{1 2 4})$ \\
\hline Males - no. (\%) & $72(58.1 \%)$ \\
Females - no. (\%) & $52(41.9 \%)$ \\
Age ( \pm SD) & $50.1 \pm 16.9$ years \\
COM, type - no. (\%) & \\
- OMCC & $53(42.8 \%)$ \\
- OMCS & $66(53.2 \%)$ \\
- OMCC + OMCS & $5(4.0 \%)$ \\
Affected ear(s) - no. (\%) & $56(45.2 \%)$ \\
- right & $40(32.2 \%)$ \\
- left & $28(22.6 \%)$ \\
- both & $82(66.1 \%)$ \\
Previous surgery for COM - no. (\%) & $42(33.9 \%)$ \\
- yes & \\
\hline
\end{tabular}

COM, chronic otitis media; OMCS, otitis media chronica simplex; OMCC otitis media chronica cholesteatomatosa.

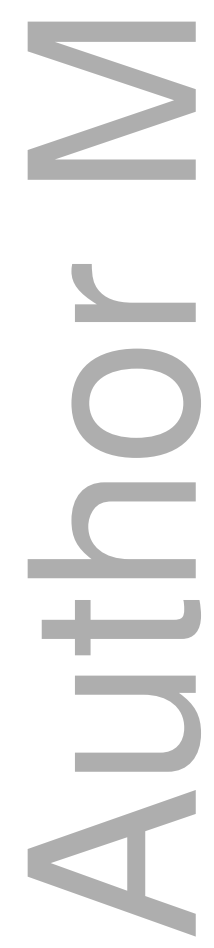

This article is protected by copyright. All rights reserved 
Table 2. Descriptive statistics of the ZCMEI-21-E single items.

\begin{tabular}{|c|c|c|c|c|}
\hline & mean & $\min -\max$ & ITC & $\alpha$ \\
\hline I. ear signs and symptoms & & & & 0.81 \\
\hline 1. ear pain & 0.68 & $0-4$ & 0.607 & \\
\hline 2. discharge & 0.73 & $0-4$ & 0.461 & \\
\hline 3. itching & 1.04 & $0-4$ & 0.578 & \\
\hline 4. feeling of pressure & 1.06 & $0-4$ & 0.421 & \\
\hline 5. balance & 0.77 & $0-4$ & 0.530 & \\
\hline II. hearing & & & & 0.83 \\
\hline 6. tinnitus & 1.63 & $0-4$ & 0.497 & \\
\hline 7. hearing (filter question) & 1.88 & $0-4$ & 0.662 & \\
\hline 8. when many people speak at the same time & 1.69 & $0-4$ & 0.618 & \\
\hline 9. telephone, alarm-clock & 1.34 & $0-4$ & 0.535 & \\
\hline 10. fear of not hearing other people & 2.04 & $0-4$ & 0.661 & \\
\hline III. psychosocial impact & & & & 0.88 \\
\hline 11. impact of ear symptoms on HRQoL & 1.79 & $0-4$ & 0.742 & \\
\hline 12. protection from water & 1.06 & $0-4$ & 0.647 & \\
\hline 13. activities with family and friends & 1.32 & $0-4$ & 0.717 & \\
\hline 14. in public (e.g. occupation, shopping) & 1.27 & $0-4$ & 0.657 & \\
\hline 15. making contact with other people & 0.88 & $0-4$ & 0.672 & \\
\hline 16. quality of sleep & 1.12 & $0-4$ & 0.579 & \\
\hline 17. sadness & 2.11 & $0-4$ & 0.584 & \\
\hline 18. fear that the ear problems may persist & 1.67 & $0-4$ & 0.393 & \\
\hline IV. medical resources & & & & 0.69 \\
\hline 19. medical consultations & 1.07 & $0-4$ & 0.524 & \\
\hline 20. antibiotics (oral) & 0.68 & $0-4$ & 0.333 & \\
\hline 21. ear drops & 0.97 & $0-4$ & 0.185 & \\
\hline
\end{tabular}

a, Cronbach's $\alpha$ for individual subscales; ITC, item-total-correlation; min, lowest value; max, highest value. 
Table 3. Comparison and correlation of total scores and subscale scores of the ZCMEI-21, ZCMEI-21-E and the EQ-5D-5L.

\begin{tabular}{|c|c|c|}
\hline & $\begin{array}{l}\text { Current study, English, } \\
\text { ZCMEI-21-E }(n=124)\end{array}$ & $\begin{array}{l}\text { Original German validation } \\
\text { study, ZCMEl-21 }(n=76)\end{array}$ \\
\hline ZCMEI-21-E/ZCMEI-21 total score ( \pm SD) & $25.9 \pm 15.8$ & $29.7 \pm 16.1$ \\
\hline I. Ear signs and symptoms & $4.2 \pm 3.9$ & $5.1 \pm 3.9$ \\
\hline II. Hearing & $8.2 \pm 5.1$ & $8.5 \pm 5.2$ \\
\hline III. Psychosocial impact & $10.9 \pm 7.9$ & $13.1 \pm 7.9$ \\
\hline IV. Medical resources & $2.6 \pm 2.4$ & $3.0 \pm 2.3$ \\
\hline \multicolumn{3}{|l|}{ EQ-5D-5L $( \pm$ SD) } \\
\hline - descriptive system score & $0.80 \pm 0.23$ & $0.92 \pm 0.14$ \\
\hline - VAS score & $79.0 \pm 19.7$ & NA \\
\hline \multicolumn{3}{|l|}{ total score correlation $^{\dagger}$} \\
\hline $\begin{array}{l}- \text { to question directly assessing } \\
\text { HRQoL }\end{array}$ & $r=0.67, p<0.0001$ & $r=0.74, p<0.0001$ \\
\hline $\begin{array}{l}\text { - to EQ-5D-5L descriptive system } \\
\text { score }\end{array}$ & $r=0.55, p<0.0001$ & $r=0.60, p<0.0001$ \\
\hline
\end{tabular}

${ }^{\dagger}$ Spearman's rank correlation coefficient and p-value. 


\section{ZCMEI-21}

German

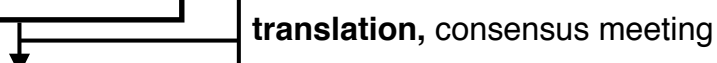

ZCMEI-21-E

v1

$$
\text { cognitive debriefing } 1(n=5)
$$

ZCMEI-21-E

v2

back translation, review of the back

translation against the original version

\section{ZCMEI-21-E}

v3

\section{cognitive debriefing $2(n=5)$}

ZCMEI-21-E

final version
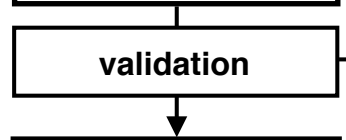

ZCMEI-21-E

validated
ZCMEI-21-E, completed by COM patients $(n=124)$ in 4 study centers

inclusion criteria:

- chronic otitis media

- sufficient English language skills

- minimum age: 18 years included patients $(n=124)$ 
A ${ }^{20}{ } r=0.58, p<0.0001 \quad$ coa_132__ff4.p20f $r=0.55, p<0.0001$

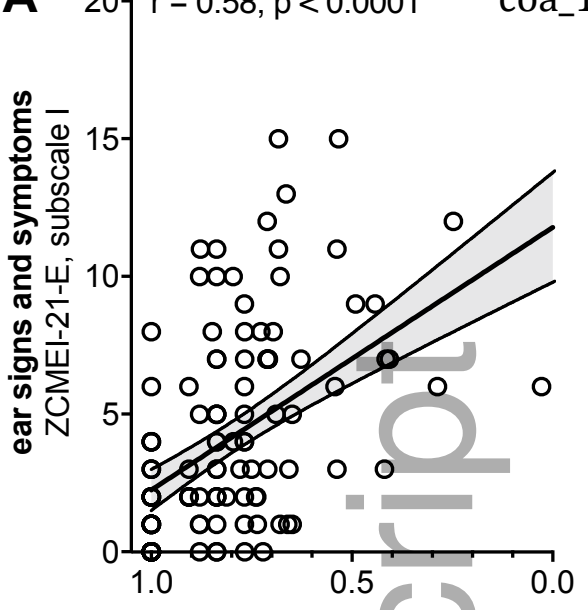

EQ-5D descriptive system score

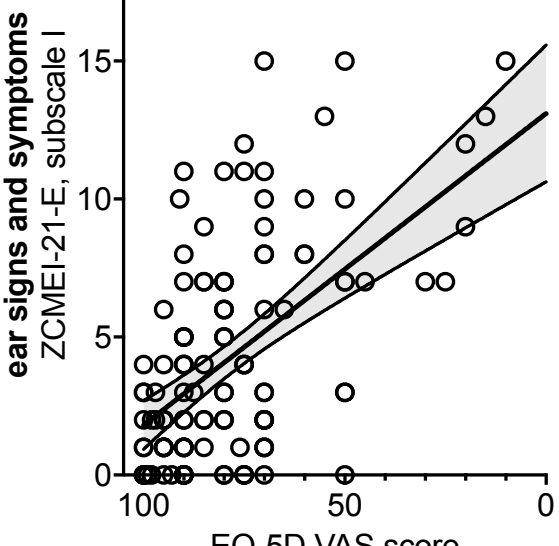

B ${ }^{20} 7^{r=0.47, p<0.00010}$

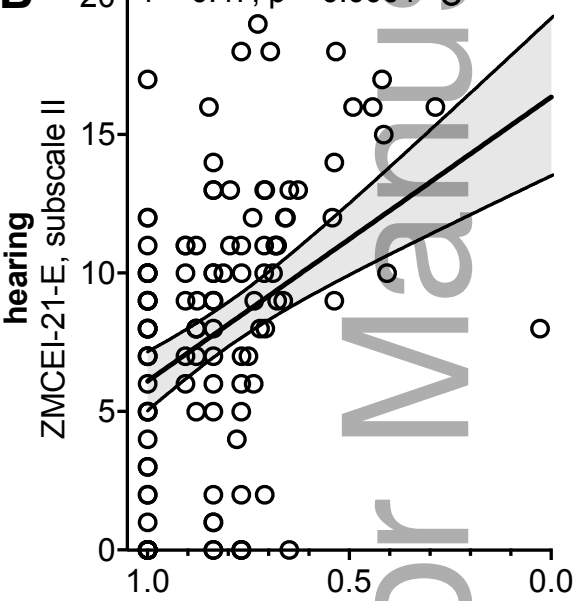

EQ-5D descriptive system score

E $\left.\quad{ }^{25}\right] r=0.47, p<0.0001$

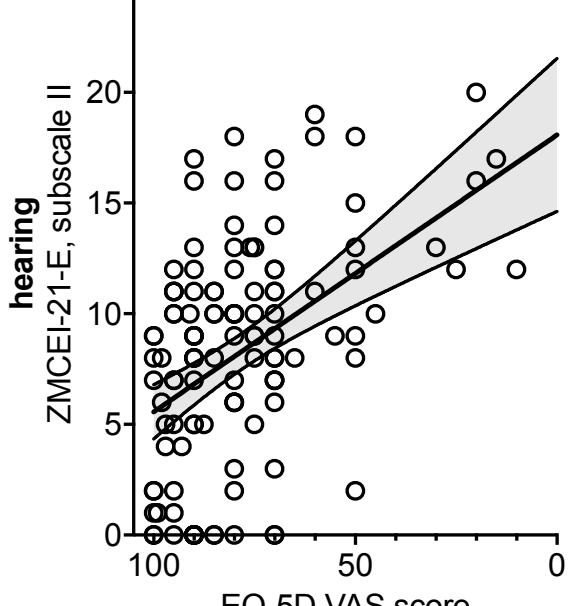

EQ-5D VAS score

F $\left.\quad{ }^{35}\right]^{r=0.51, p<0.0001}$

C $\quad{ }^{35}{ } r=0.63, p<0.0001$

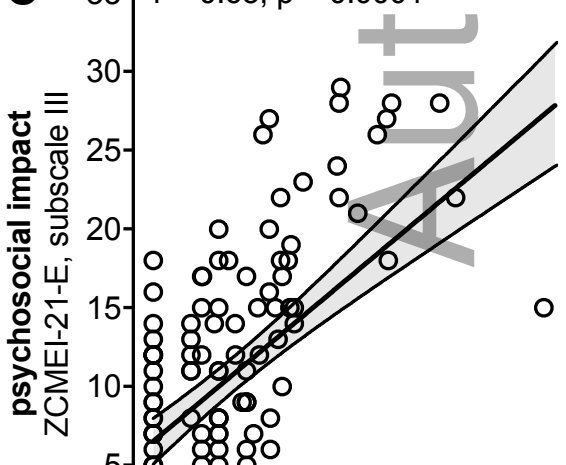

$5-8,888$

18008
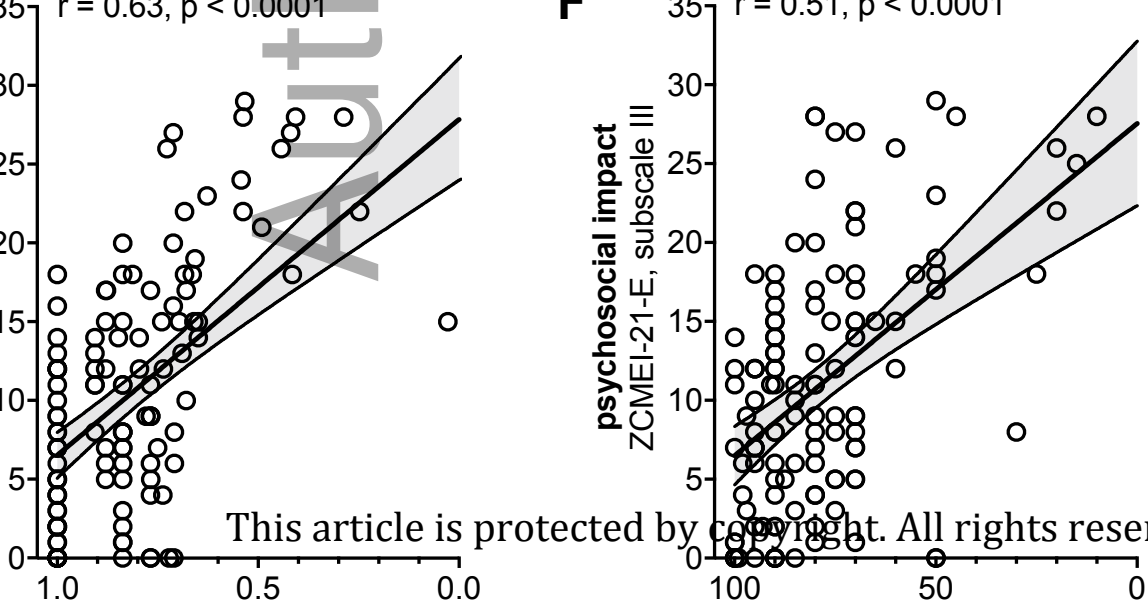


\section{University Library}

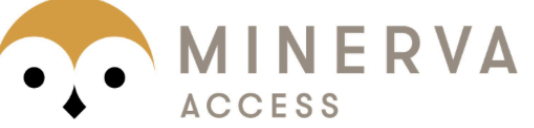

A gateway to Melbourne's research publications

Minerva Access is the Institutional Repository of The University of Melbourne

\section{Author/s:}

Chatzimichalis, M;Epprecht, L;Weder, S;Shaul, C;Folchert, KJE;Machala, MC;Goosmann, MM;Naville, M;Zhu, A;Roeoesli, C;Lee, DJ;Cass, SP;Briggs, R;Huber, AM;Baechinger, D

Title:

English translation and validation of the Zurich chronic middle ear inventory (ZCMEI-21-E) assessing quality of life in chronic otitis media: A prospective international multicentre study

Date:

2019-05-01

\section{Citation:}

Chatzimichalis, M., Epprecht, L., Weder, S., Shaul, C., Folchert, K. J. E., Machala, M. C., Goosmann, M. M., Naville, M., Zhu, A., Roeoesli, C., Lee, D. J., Cass, S. P., Briggs, R., Huber, A. M. \& Baechinger, D. (2019). English translation and validation of the Zurich chronic middle ear inventory (ZCMEI-21-E) assessing quality of life in chronic otitis media: A prospective international multicentre study. CLINICAL OTOLARYNGOLOGY, 44 (3), pp.254-262. https://doi.org/10.1111/coa.13275.

Persistent Link:

http://hdl.handle.net/11343/285278 